\title{
OPEN Real-world outcomes versus clinical trial results of immunotherapy in stage IV non-small cell lung cancer (NSCLC) in the Netherlands
}

\author{
Christine M. Cramer-van der Welle ${ }^{1 凶}$, Marjon V. Verschueren ${ }^{2}$, Merel Tonn $^{2}$, \\ Bas J. M. Peters ${ }^{2}$, Franz M. N. H. Schramel ${ }^{3}$, Olaf H. Klungel ${ }^{4}$, Harry J. M. Groen ${ }^{5}$, \\ Ewoudt M. W. van de Garde ${ }^{2,4}$ \& The Santeon NSCLC Study Group*
}

This study aims to assess how clinical outcomes of immunotherapy in real-world (effectiveness) correspond to outcomes in clinical trials (efficacy) and to look into factors that might explain an efficacy-effectiveness (EE) gap. All patients diagnosed with stage IV non-small cell lung cancer (NSCLC) in 2015-2018 in six Dutch large teaching hospitals (Santeon network) were identified and followed-up from date of diagnosis until death or end of data collection. Progression-free survival (PFS) and overall survival (OS) from first-line (1L) pembrolizumab and second-line (2L) nivolumab were compared with clinical trial data by calculating hazard ratios (HRs). From 1950 diagnosed patients, 1005 (52\%) started with any $1 \mathrm{~L}$ treatment, of which 83 received pembrolizumab. Nivolumab was started as $2 \mathrm{~L}$ treatment in 141 patients. For both settings, PFS times were comparable between real-world and trials (HR 1.08 (95\% Cl 0.75-1.55), and HR 0.91 (95\% Cl 0.74-1.14), respectively). OS was significantly shorter in realworld for $1 \mathrm{~L}$ pembrolizumab (HR 1.55; 95\% Cl 1.07-2.25). Receiving subsequent lines of treatment was less frequent in real-world compared to trials. There is no EE gap for PFS from immunotherapy in patients with stage IV NSCLC. However, there is a gap in OS for $1 \mathrm{~L}$ pembrolizumab. Fewer patients proceeding to a subsequent line of treatment in real-world could partly explain this.

For patients with stage IV non-small cell lung cancer (NSCLC), immune checkpoint inhibitors (ICIs) have demonstrated promising results in disease progression and survival in randomized clinical trials (RCTs) ${ }^{1-6}$. Currently, there are three FDA and EMA approved programmed cell death protein-1/programmed cell death ligand-1 (PD-1/PD-L1) inhibitors available for treatment of stage IV NSCLC. These include nivolumab (antiPD-1), pembrolizumab (anti-PD-1), and atezolizumab (anti PD-L1) ${ }^{1}$. However, results from RCTs are not easily generalizable to clinical practice due to strict eligibility criteria ${ }^{7-9}$. While the population is ageing, which is associated with an increased risk of lung cancer, most patients that are included in RCTs are under 65 years old ${ }^{10,11}$. Additionally, NSCLC patients with comorbidities, especially auto-immune diseases, or poor performance status are often not eligible for participating in RCTs ${ }^{11,12}$. At present, RCTs are still the gold standard to prove efficacy for new oncology therapies ${ }^{7,8}$. Consequently, there could be a gap between the efficacy demonstrated in RCTs and the effectiveness of systemic therapies in clinical practice.

Recently, we have demonstrated that survival of patients with metastatic NSCLC treated with chemotherapy or targeted therapy is nearly one-quarter shorter in real-world practice than for patients included in trials ${ }^{13}$. For immunotherapy in metastatic NSCLC, some efforts have been made as well to provide insight into this possible gap. So far, most studies reported comparable outcomes in real-world compared to those observed in clinical trials. For example, a recent study from The Netherlands by Smit et al. ${ }^{14}$ assessed real-world effectiveness of ICIs in a Dutch population of patients with advanced NSCLC and showed similar median survival times as those

\footnotetext{
${ }^{1}$ Santeon Hospital Group, Santeon, Herculesplein 38, 3584 AA Utrecht, The Netherlands. ${ }^{2}$ Department of Clinical Pharmacy, St. Antonius Hospital, Utrecht, Nieuwegein, The Netherlands. ${ }^{3}$ Department of Pulmonary Diseases, St. Antonius Hospital, Utrecht, Nieuwegein, The Netherlands. ${ }^{4}$ Division of Pharmacoepidemiology and Clinical Pharmacology, Department of Pharmaceutical Sciences, Utrecht University, Utrecht, The Netherlands. ${ }^{5}$ Department of Pulmonary Diseases, University Medical Center Groningen, University of Groningen, Groningen, The Netherlands. *A list of authors and their affiliations appears at the end of the paper. ${ }^{\otimes}$ email: c.van.der.welle@antoniusziekenhuis.nl
} 
of phase III studies, except for patients with Eastern Cooperative Oncology Group performance status (ECOG PS) of $\geq 2$. The latter study, however, did not discriminate between type of drug nor compared survival curves to detect potential differences between survival dynamics in real-world and trials. Many studies have shown that the shape of the survival curve for immunotherapy is different than for chemotherapy, with some extra early deaths with immunotherapy and a plateau of long survivors later on. Furthermore, patient characteristics have not been compared with trial cohorts in search for explanatory factors for any efficacy-effectiveness gap.

The aim of this study is to assess how clinical outcomes of specific immunotherapies for patients with stage IV NSCLC in real-world (effectiveness) correspond to the results from clinical trials (efficacy) and to look into factors that might explain an efficacy-effectiveness gap.

\section{Patients and methods}

Data sources. Clinical data from six hospitals of the Santeon hospital network were used for this study. This hospital network consists of seven large (non-university) teaching hospitals that are geographically spread over The Netherlands and serve $>11 \%$ of the Dutch population ${ }^{15}$. Patients diagnosed with stage IV NSCLC were identified from the Netherlands Cancer Registry (NCR) ${ }^{16}$. Data on diagnosis, vital status, and patient characteristics were obtained from the NCR.

Data on pharmacotherapy from the Santeon Farmadatabase was merged with the NCR dataset, to construct an overview of all applied regimes in the study population. The Santeon Farmadatabase contains, among others, information on individual prescriptions with drug name, dosage, date of administration and administration route $^{17}$. Patients' medical records were used to complement and validate the dataset.

Data was collected and managed with REDCap ${ }^{18}$, hosted at St. Antonius Hospital, Utrecht/Nieuwegein, the Netherlands.

Study population. Patients with stage IV NSCLC diagnosed between January 1st, 2015 and December 31st, 2018 in one of six Santeon Hospitals were selected. Staging was based on the 7th edition TNM classification for the years 2015-2016 and 8th edition for incidence years 2017-2018. Characteristics that were recorded for included patients were age (at year of diagnosis), gender, ECOG PS, histology, brain metastases, pre-existing autoimmune disease, and PD-L1 expression of the tumour. Also, the type of treatment (best supportive care (BSC), chemotherapy, targeted therapy or immunotherapy), line of treatment, hospital where the patient was treated, and (in case of immunotherapy) information on immune related adverse events (irAE) and palliative radiotherapy were collected.

Identification of systemic treatments per patient. First-line treatment was defined as the initial systemic treatment that was started after diagnosis. Second-line or further line treatment was defined as systemic treatment applied after completion or discontinuation because of disease progression of first or second-line treatment, respectively. After the identification of all different regimens we ordered patients in four different categories: chemotherapy, treatment with tyrosine kinase inhibitors (TKIs), immunotherapy, or best supportive care. For this study we focussed on immunotherapy and identified the most frequently used types of drugs (pembrolizumab and nivolumab) in first and second-line in our database. Second-line nivolumab for NSCLC in the Netherlands was introduced in March 2016. In 2017, first-line pembrolizumab for NSCLC with PD-L1 tumour proportion score (TPS) $\geq 50 \%$ was introduced.

Reference outcome. After identification of the most commonly used types of immunotherapy, corresponding reference outcomes were established from clinical trials. These clinical trials were identified by a literature search on PubMed for clinical trials used for approval of immunotherapy drugs. If multiple registration studies were published with different patient populations, the study with the most comparable patient population (based on the distribution of stage, PD-L1 expression, and histology) to our cohort was chosen. We searched for updated publications of the selected studies (if applicable) to use up-to-date data and to utilize as much as possible of their follow-up time for the comparison with real-world data.

Statistical analysis. All statistical analyses were conducted using R software package version 3.6.1.

To present an overview of baseline characteristics for all treated patients, frequencies (proportions) were calculated for categorical variables, and means (standard deviations) were provided for normally distributed continuous data.

For patients who received immunotherapy in first or second-line, overall survival (OS) was calculated as time between start date of treatment until date of death from any cause. Patients still alive at January 1, 2020 were censored as this was the end of follow-up date. Progression free survival (PFS) was calculated as time between start date of treatment until the occurrence of progression according to RECIST criteria when noted. Date of death was noted in absence of acknowledged progression from the individual patient files. Survival curves were obtained for the treatment groups using the Kaplan-Meier method.

The potential existence of an efficacy-effectiveness gap was assessed in two manners. First, a so-called efficacyeffectiveness (EE) factor was calculated by dividing the patient's individual median survival by the corresponding reference OS and PFS from clinical trials ${ }^{13}$. This factor was used to estimate the presence of an efficacy-effectiveness gap and shows how the patient's individual survival is related to survival presented in the reference RCT. As an example, an EE factor of 0.70 shows that median survival is $30 \%$ shorter in clinical practice than in RCTs. The Wilcoxon signed rank test was used to analyse the distribution of the EE factors. The null hypothesis (median OS in real-world is similar to median OS reported in clinical trials) is rejected if the distribution is significantly different from test value 1.0. 


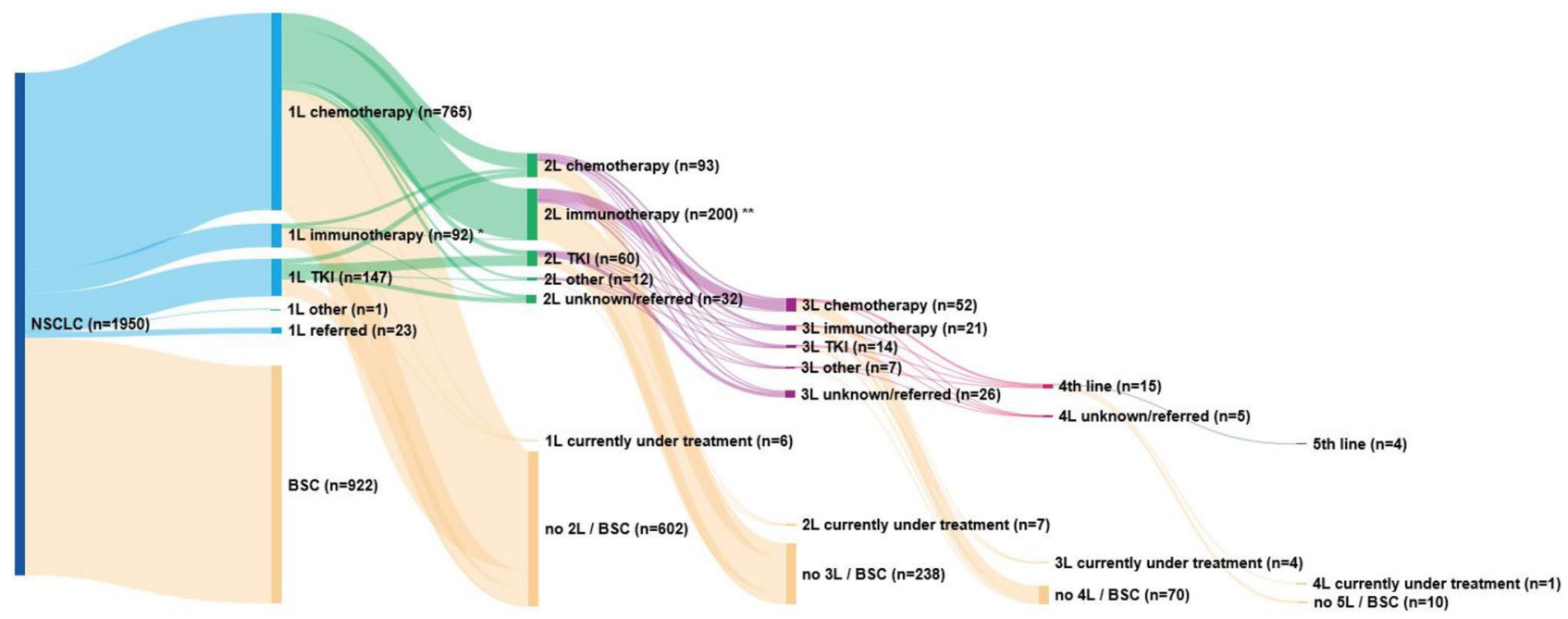

Figure 1. Treatment patterns of patients diagnosed with stage IV NSCLC between 2015 and 2018 in six Dutch hospitals. BSC, best supportive care; TKI, Tyrosine Kinase Inhibitors. ${ }^{\star} 1 \mathrm{~L}$ immunotherapy: pembrolizumab: $\mathrm{n}=83$ (90\%), nivolumab: $\mathrm{n}=3(3 \%)$, other $\mathrm{n}=6(7 \%)$. ${ }^{*} 2 \mathrm{~L}$ immunotherapy: nivolumab: $\mathrm{n}=164(82 \%$, of which $\mathrm{n}=141$ with non-squamous histology), pembrolizumab: $\mathrm{n}=20(10 \%)$, atezolizumab: $\mathrm{n}=13$ (6.5\%), other: $\mathrm{n}=3$ (1.5\%).

Second, we assessed hazard ratios (HRs) between real-world immunotherapy treatment regimens and groups in corresponding RCTs to compare OS and PFS. This was achieved by first digitizing the Kaplan-Meier (KM) curves for the immunotherapy arm from the included clinical trials with the R-package 'digitize. The extracted data points for survival probability were used for reconstructing the KM curve with the algorithm as described by Guyot et al. ${ }^{19}$ The coordinates of the KM curve from the published graph were read in with the R-coding script by Guyot et al., together with the information on numbers at risk and total number of events, to reconstruct the KM data. With this reconstructed individual patient data (IPD), KM curves and Cox HRs were estimated using the $\mathrm{R}$ routines 'survfit' and 'coxph'.

Univariate and multivariate analyses for potential prognostic factors for PFS and OS in real-world were performed using Cox proportional hazards regression models. The factors with $p<0.05$ on univariate analysis were included in the multivariate analysis. In this analysis, missing values were imputed by single stochastic regression imputation (single run with all available characteristics in the model). Pearson's chi-square tests were performed to test if the proportion of the prognostic factor in real-world differed from reference RCT data, if applicable.

Ethical statement. All methods were carried out in accordance with relevant guidelines and regulations. The study was approved by the Santeon institutional review board, and all clinical information was provided in a de-identified fashion and informed consent was waived (SDB219-008). The study was performed in accordance with the ethical standards of the institutional and national research committee and with the 1964 Helsinki declaration and its later amendments or comparable ethical standards.

\section{Results}

Baseline characteristics. We identified 1950 patients diagnosed with stage IV NSCLC in the period 2015 to 2018. Figure 1 provides an overview of the different treatment patterns of all patients towards best supportive care (BSC). Of these patients, 922 (47\%) did not receive active anti-tumour treatment in 1L because of their ECOG PS, comorbidities, or at request of the patient. Twenty-three patients (1\%) were referred to other hospitals after diagnosis of whom we did not have information on treatment. Of all diagnosed patients, 1005 (52\%) received $1 \mathrm{~L}$ treatment of which $92(9 \%)$ received immunotherapy in first-line. Median OS for patients receiving chemotherapy, immunotherapy or TKI was 7.5 months, 15.6 months or 15.5 months, respectively (Appendix SFigure 1).

Of all treated patients, only $365(36 \%)$ received subsequent treatment in one of the six hospitals of which 200 $(55 \%)$ received immunotherapy. The most frequently received $1 \mathrm{~L}$ immunotherapy was pembrolizumab $(\mathrm{n}=83$, $90 \%$ of all patients treated with immunotherapy in $1 \mathrm{~L}$ ) and in second-line nivolumab for patients with nonsquamous tumour histology $(\mathrm{n}=141,71 \%)$. Based on these subgroups, two registration studies (Checkmate 057 and KEYNOTE- $024^{2,5}$ ) were identified for the comparison with real-world data.

Table 1 shows patient characteristics of patients with $1 \mathrm{~L}$ pembrolizumab or 2L nivolumab (both monotherapy). The median age of patients with $1 \mathrm{~L}$ pembrolizumab was 66 years and almost all patients (96\%) had an ECOG PS of 0 or 1 . The median age of patients with $2 \mathrm{~L}$ nivolumab was 64 years and $95 \%$ had an ECOG PS of 0 or 1 . 


\begin{tabular}{|c|c|c|c|c|}
\hline & \multicolumn{2}{|c|}{ Pembrolizumab 1st line } & \multicolumn{2}{|c|}{ Nivolumab 2nd line } \\
\hline & \begin{tabular}{|l|} 
Real-world \\
\end{tabular} & RCT $^{5}$ & Real-world & $\mathrm{RCT}^{2}$ \\
\hline Subjects & 83 & 154 & 141 & 292 \\
\hline Male & $45(54 \%)$ & $92(60 \%)$ & $74(53 \%)$ & $151(52 \%)$ \\
\hline Age (median (range)) & $66.0(35-84)$ & $64.5(33-90)$ & $64.0(44-80)$ & $61.0(37-84)$ \\
\hline \multicolumn{5}{|l|}{ ECOG PS } \\
\hline 0 & $30(36 \%)$ & $54(35 \%)$ & $44(31 \%)$ & $84(29 \%)$ \\
\hline 1 & $50(60 \%)$ & $99(64 \%)$ & $90(64 \%)$ & $208(71 \%)$ \\
\hline 2 & $3(4 \%)$ & $1(1 \%)$ & $7(5 \%)$ & - \\
\hline \multicolumn{5}{|l|}{ Histology } \\
\hline Squamous & $11(13)$ & $29(19 \%)$ & - & - \\
\hline Non-squamous & $72(87)$ & $125(81 \%)$ & $141(100 \%)$ & $292(100.0)$ \\
\hline \multicolumn{5}{|l|}{ PD-L1 expression } \\
\hline$<1 \%$ & - & - & $33(23 \%)$ & $108(37 \%)$ \\
\hline $1-49 \%$ & - & - & $29(21 \%)$ & $123^{\star}(42 \%)$ \\
\hline$\geq 50 \%$ & $83(100 \%)$ & $154(100 \%)$ & $10(7 \%)$ & \\
\hline Unknown $^{* * *}$ & - & - & $69(49 \%)$ & $61(21 \%)$ \\
\hline CNS metastases & $16(19 \%)$ & $18(12 \%)$ & $25(18 \%)$ & $34(12 \%)$ \\
\hline Subsequent systemic therapy & $14(17 \%)$ & $56(36 \%)^{* *}$ & $40(28 \%)$ & $123(42 \%)$ \\
\hline
\end{tabular}

Table 1. Patient characteristics of stage IV NSCLC patients with $1 \mathrm{~L}$ pembrolizumab or 2L nivolumab. ECOG PS, Eastern Cooperative Oncology Group Performance Status; CNS, central nervous system; RCT, randomized clinical trial. ${ }^{\star} \mathrm{PD}-\mathrm{L} 1$ expression of $\geq 1 \%$. ${ }^{\star \star}$ Including surgery and radiation therapy. ${ }^{\star \star \star}$ Not performed in realworld; not quantifiable from $\mathrm{RCT}^{2}$.

\begin{tabular}{|c|c|c|c|}
\hline & $\begin{array}{l}\text { Real-world } \\
\text { months (95\% CI) }\end{array}$ & $\begin{array}{l}\text { Clinical trial } \\
\text { months }(95 \% \mathrm{CI})\end{array}$ & $\begin{array}{l}\text { EE factor } \\
\text { median }\end{array}$ \\
\hline \multicolumn{4}{|c|}{ 1L Pembrolizumab } \\
\hline $\mathrm{mPFS}$ & $8.9(3.7-14.1)$ & $10.3(6.7-\mathrm{NR})^{5}$ & 0.85 \\
\hline $\mathrm{mOS}$ & $15.8(9.4-22.1)$ & $30.0(18.3-\mathrm{NR})^{33}$ & $0.45^{\mathrm{a}}$ \\
\hline \multicolumn{4}{|c|}{ 2L Nivolumab } \\
\hline mPFS & $3.8(3.0-4.7)$ & $2.3(2.2-3.4)^{34}$ & $1.61^{\mathrm{a}}$ \\
\hline $\mathrm{mOS}$ & $8.2(5.9-10.6)$ & $12.2(9.7-15.1)^{34}$ & 0.65 \\
\hline
\end{tabular}

Table 2. Median survival in real-world clinical practice and randomized clinical trials. mPFS, median progression-free survival; mOS, median overall survival; EE factor, efficacy-effectiveness factor. ${ }^{\text {a Significantly }}$ different ( $p$-values $<0.05)$ from test value 1.00 (one-sample Wilcoxon signed-rank test).

Survival. Median PFS and OS for 1L pembrolizumab treatment were 8.9 and 15.8 months, respectively. For patients treated with 2L nivolumab, median PFS was 3.8 and median OS was 8.2 months (Table 2). One-year survival was $57 \%$ for $1 \mathrm{~L}$ pembrolizumab and $42 \%$ for $2 \mathrm{~L}$ nivolumab. In the corresponding clinical trials on pembrolizumab and nivolumab, one-year survival was $70 \%$ and $51 \%$, respectively ${ }^{2,20}$.

EE factor analysis. Median OS was shorter for all patients who received $1 \mathrm{~L}$ pembrolizumab in real-world practice compared to the clinical trial. Table 2 shows an EE factor of 0.45 ( $p<0.001$ from 1 ), which means that median survival is $55 \%$ shorter for patients treated in clinical practice relative to median survival from the registration clinical trial. There was no significant difference in median PFS for 1L pembrolizumab, with an EE factor of 0.85 ( $p=0.86)$.

For 2L nivolumab, the EE factor for median OS is $0.65(p=0.065)$. For PFS, we found an EE factor of 1.61 $(p<0.001)$ (Table 2). This indicates that median PFS is $61 \%$ higher for nivolumab in clinical practice, compared to median PFS from the RCT.

Proportional hazard analysis. The estimated HRs for $1 \mathrm{~L}$ pembrolizumab in real-world compared to data from the RCT were 1.08 (95\% CI 0.75-1.55) and 1.55 (95\% CI 1.07-2.25) for PFS and OS, respectively (Fig. 2). For 2L nivolumab the estimated HRs were 0.91 (95\% CI 0.74-1.14) for PFS and 1.17 (95\% CI 0.93-1.48) for OS (Fig. 3). This indicates that PFS is comparable between real-world and RCTs, and in real-world OS is significantly shorter for $1 \mathrm{~L}$ immunotherapy.

From the univariate cox proportional hazards regression model, no significant associations were found with PFS or OS for all potential prognostic factors in real-world patients with $1 \mathrm{~L}$ pembrolizumab, or for PFS in realworld patients with $2 \mathrm{~L}$ nivolumab. Higher ECOG PS $(0-1 \mathrm{vs} . \geq 2)$ at start of $2 \mathrm{~L}$ nivolumab was associated with 

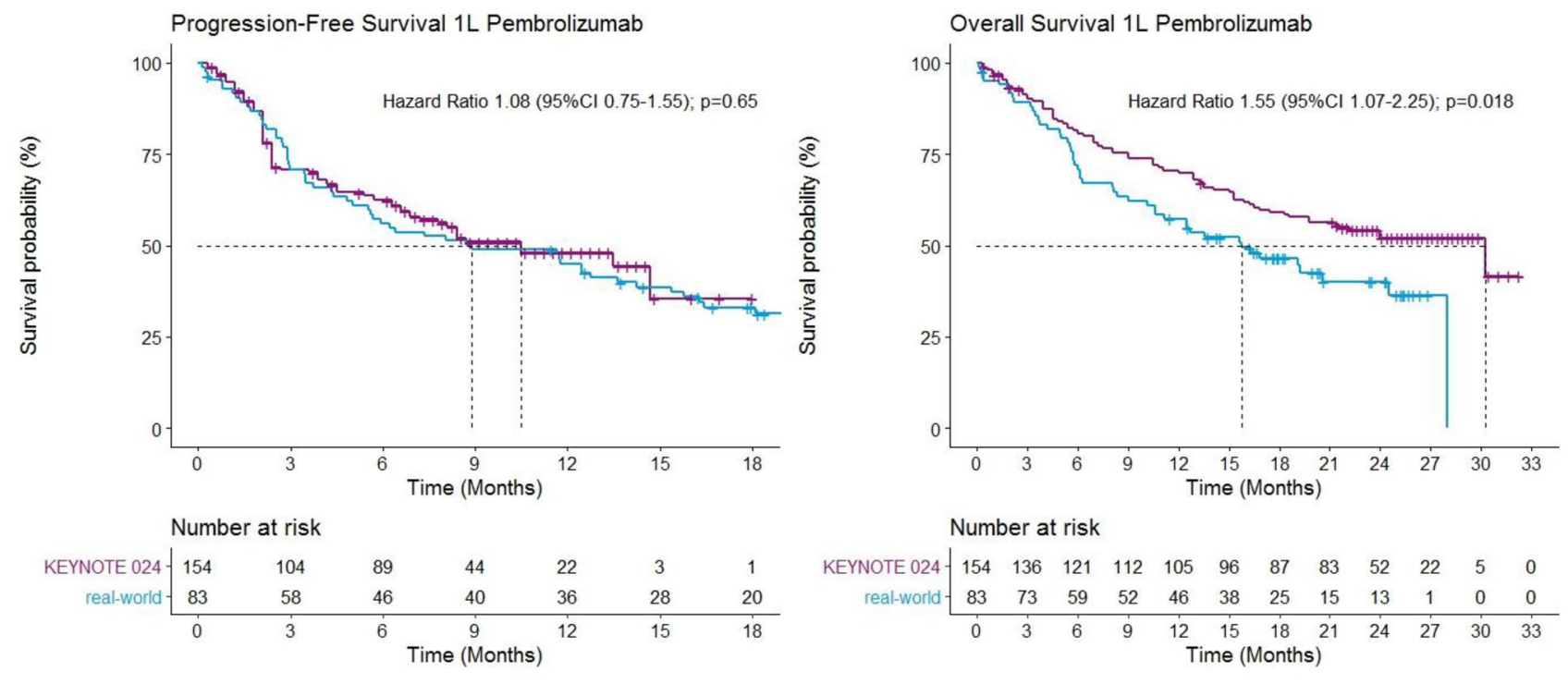

- KEYNOTE 024 * real-world

- KEYNOTE 024 * real-world

Figure 2. Kaplan-Meier curves of PFS and OS in patients receiving $1 \mathrm{~L}$ pembrolizumab in real-world and clinical trial.
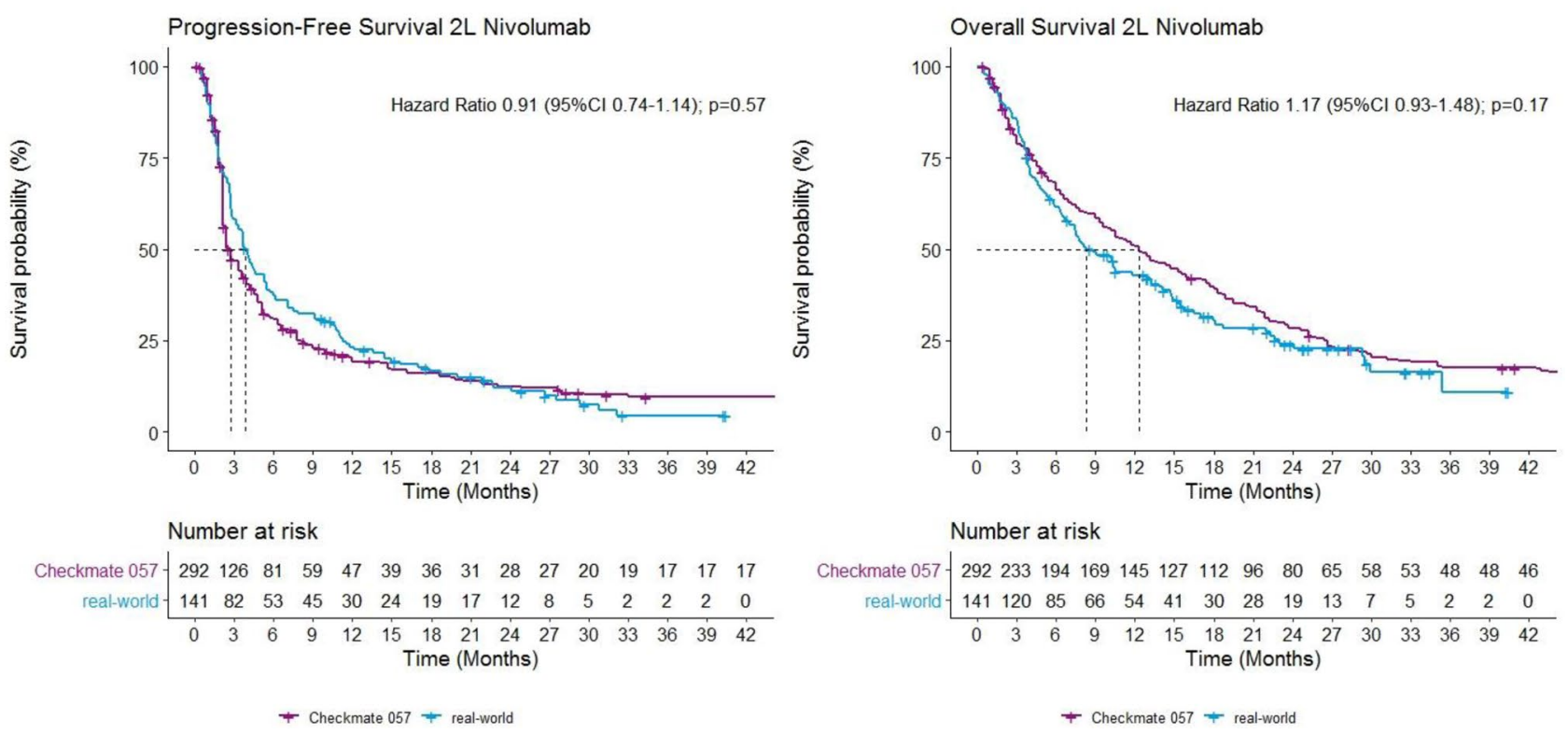

Figure 3. Kaplan-Meier curves of PFS and OS in patients receiving $2 \mathrm{~L}$ nivolumab in real-world and clinical trial.

shorter OS (HR 2.23; 95\% CI 1.03-4.83; $p=0.043$ ). Further analysis showed that the proportion of patients with ECOG PS $\geq 2$ in real-world is higher compared to patients in clinical trials ( $5 \%$ versus $0 \%, p<0.001)$.

When looking at explanatory factors after starting $1 \mathrm{~L}$ pembrolizumab in real-world, we found that PFS was influenced by the occurrence of irAEs. Having an irAE reduces the hazard by a factor of 0.48 (95\% CI 0.26-0.89; $p=0.019$ ). For $2 \mathrm{~L}$ nivolumab, the occurrence of irAEs was also an explanatory factor for longer PFS and OS (HR 0.45 ; 95\% CI 0.30-68; $p<0.001$ and HR 0.44; 95\% CI 0.27-0.70; $p<0.001$, respectively).

In real-world, the proportion of patients with irAEs was significantly lower compared to clinical trials for both $1 \mathrm{~L}$ pembrolizumab and $2 \mathrm{~L}$ nivolumab (31\% versus $77 \%$ with $p<0.001$, and $28 \%$ versus $69 \%$ with $p<0.001$, respectively). Furthermore, fewer patients received a subsequent line of treatment in real-world compared to patients in clinical trials after both $1 \mathrm{~L}$ pembrolizumab and $2 \mathrm{~L}$ nivolumab $(17 \%$ versus $36 \%$ with $p=0.002$, and $28 \%$ versus $42 \%$ with $p=0.014$, respectively). 


\section{Discussion}

This study showed that PFS of patients with stage IV NSCLC treated with immunotherapy is comparable between real-world and clinical trials. However, OS is significantly shorter for patients with $1 \mathrm{~L}$ pembrolizumab in realworld (median 15.8 vs. 30 months; HR 1.55 (95\% CI 1.07-2.25)). This finding is in line with our previous research ${ }^{13}$ which showed that survival after first-line chemotherapy and targeted therapy is around one quarter shorter in real-world compared to clinical trial data. The present study extends this finding to first-line pembrolizumab monotherapy in patients with $\geq 50 \%$ PD-L1 expression.

To our knowledge, this is the first study that calculated hazard ratios for PFS and OS of real-world versus clinical trials. This approach provided insight that stage IV NSCLC patients who are treated with immunotherapy in regular clinical practice have a comparable period of PFS time but that this does not extend to a similar OS benefit as demonstrated in the registration trial. This implies that something differs between regular practice and clinical trial participants after progression on immunotherapy. One explanation from our data could be the receiving of a next line of treatment being more frequent in clinical trial participants compared to in real-world (two times more frequent in $1 \mathrm{~L}$ setting, and 1.5 times in $2 \mathrm{~L}$ setting). Patients who received immunotherapy through participating in a clinical trial, for example, might have an above average intrinsic willingness to search for further treatment options after treatment failure. Other possible explanations could be that clinics active in trial enrolment communicate more about remaining experimental treatments, or that patients characteristics, other than performance status, limit the tolerability for subsequent systemic treatment (in this case chemotherapy) after progression on immunotherapy to a larger extent in regular care patients.

The results on median OS and PFS as found in our study are largely in line with previous observational studies on $1 \mathrm{~L}$ immunotherapy treatment with pembrolizumab in real-world. Velcheti et al.$^{20}$ evaluated real-world survival in patients with metastatic NSCLC with an PD-L1 expression of $\geq 50 \%$ and ECOG PS $\leq 1$, and found a median PFS of 6.8 months and median OS of 19.1 months. Their PFS and OS are slightly shorter than found in the KEYNOTE-024 clinical trial, but real-world OS is longer than was found in our study, which could be explained by only including patients with ECOG PS 0 or 1 , or by the higher percentage of patients with secondline systemic therapy as compared to our cohort (28\% vs. $17 \%$, respectively). A French study which included patients with advanced NSCLC and PD-L1 expression of $\geq 50 \%$, reported a median PFS of 10.1 months and a median OS of 15.2 months for patients treated with pembrolizumab in first-line, including patients with brain metastases and ECOG PS $2^{21}$. As in the results of the current study, their PFS is comparable with the median PFS from the KEYNOTE-024 trial of 10.3 months, whereas OS is shorter (comparable to our real-world findings). Tambo et al. ${ }^{22}$ found a median of 6.1 for PFS which is shorter than the median PFS from both the registration study and the current study. Shorter PFS may be explained by inclusion of patients with ECOG PS 2 (12\%) and 3-4 (11\%). Their OS did not reach the median and therefore could not be compared.

The outcomes found for $2 \mathrm{~L}$ nivolumab are also in line with previous studies with real-world data. Crinò et al. ${ }^{23}$ found a median PFS for patients treated with nivolumab in second or further line of 4.2 months and a median OS of 7.9 months, which is comparable with the present findings ( 3.8 and 8.2 months, respectively). Another study in Dutch patients treated with second-line nivolumab reported a median PFS and OS of 2.6 and 10.0 months, respectively ${ }^{24}$. Grossi et al. ${ }^{25}$ reported results from the expanded access program in Italy on 1588 patients, including patients with ECOG PS 2 and aged $\geq 75$ years, where median PFS was 3.0 months and median OS 11.3 months. The results of those three studies on real-world nivolumab in second-line suggest that results for PFS and OS in real-world and RCTs are indeed comparable. Interesting from our data is that the median PFS from 2L nivolumab was significantly longer than in the clinical trial but without any difference in overall hazard (HR 0.91). An explanation could be a difference in how date of progression is determined between the two settings. To illustrate, in Checkmate 057 disease progression was assessed nine weeks after start of treatment and every six weeks thereafter, compared to every 8 weeks in real-world. This relatively small difference in timing could lead to larger absolute differences with a median PFS in weeks range in $2 \mathrm{~L}$ settings. Besides this, our finding also highlights that comparing median PFS times only could lead to biased conclusions about relative effectiveness.

Regarding prognostic factors, for patients treated with $2 \mathrm{~L}$ nivolumab, the negative association between a higher/worse ECOG PS and OS is in line with previous research. Crinò et al. ${ }^{23}$ also found ECOG PS to be a poor prognostic factor. Additionally, the results from Schouten et al. ${ }^{24}$ from the Dutch expanded access program and the study by Martin et al. ${ }^{26}$ show similar results. A Dutch study on real-world effectiveness of ICIs in patients with stage IV NSCLC in first and second-line also shows that an ECOG PS $\geq 2$ is a poor prognostic factor ${ }^{14}$. Additionally, in a phase $3 \mathrm{~B} / 4$ community-based study of nivolumab monotherapy in previously treated patients with advanced NSCLC including patients with poor performance status (Checkmate 153), a median overall survival of 4.0 months was found in patients with ECOG PS $2^{27}$. Next to the association of ECOG PS $\geq 2$ and worse outcome for patients with $2 \mathrm{~L}$ nivolumab in real-world, the proportion of patients with ECOG PS $\geq 2$ in real-world is significantly higher compared to the clinical trial. This confirms the general thinking that trials select more fit patients, where patients with higher ECOG PS in clinical practice aim for immunotherapy as well, resulting in an EE gap.

Besides this, our data also confirms the relationship between irAEs and outcomes of immunotherapy. Similar to Lisberg et al. ${ }^{28}$ who retrospectively analysed the relationship in patients that were treated with pembrolizumab in KEYNOTE-001, we found improved survival for patients with irAEs. Another study by Haratani et al. ${ }^{29}$ also revealed that irAEs were positively associated with survival outcome in nivolumab treated patients. Ricciuti et al. ${ }^{30}$ confirmed the positive relationship between the occurrence of irAEs and survival for patients with $2 \mathrm{~L}$ nivolumab as well.

The main strengths of this study are its complete and precise data, nationwide multicentre approach, and that PFS and OS were compared both through comparison of medians as well as through a proportional hazards approach. The latter method better addresses that many patients were still alive at time of conducting the 
analyses and provides a unique opportunity to compare survival dynamics not captured by a single value for median survival.

Limitations of this study are that (patient) factors potentially responsible for an efficacy-effectiveness gap could only be analysed on the cohort level instead of patient level, because individual patient data from the respective clinical trials were not available. When available, these could have been analysed in a multivariable cox regression model potentially leading to identification of additional factors associated with the EE gap. Better identification of these factors could lead to trial designs matching real-world populations better and eventually smaller EE gaps. Besides this, another limitation is that the treatment patterns shown in Fig. 1 do not match with current practice anymore (introduction of $1 \mathrm{~L}$ immunochemotherapy combination limiting the use of $2 \mathrm{~L}$ immunotherapy). Nevertheless, this does not compromise the relative effectiveness assessments within specific treatments as conducted in our study.

Apart from the well-known complexity of how to translate progression free survival times to overall survival when survival data of the trial cohort is not mature yet ${ }^{31,32}$, the present study shows that this translation can be even more complex later on when it comes to generalizability to real-world settings. Considering that costeffectiveness assessments are often based on overall survival data from trials, our observed shorter overall survival time after $1 \mathrm{~L}$ pembrolizumab in real-world compared to the clinical trial data could provide an argument to rework the initial cost-effectiveness assessment.

\section{Conclusion}

There is no efficacy-effectiveness gap for the outcome PFS for immunotherapy in patients with stage IV NSCLC. However, there is a gap in OS for first-line pembrolizumab. Fewer patients proceeding to a subsequent line of treatment in real-world could partly explain this. PFS and OS results from clinical trials can differ in generalizability to regular clinical practice.

Received: 23 November 2020; Accepted: 25 February 2021

Published online: 18 March 2021

\section{References}

1. Herzberg, B., Campo, M. J. \& Gainor, J. F. Immune checkpoint inhibitors in non-small cell lung cancer. Oncologist 22, 81-88. https://doi.org/10.1634/theoncologist.2016-0189 (2017).

2. Borghaei, H. et al. Nivolumab versus docetaxel in advanced nonsquamous non-small-cell lung cancer. N. Engl. J. Med. 373, 1627-1639. https://doi.org/10.1056/NEJMoa1507643 (2015).

3. Brahmer, J. et al. Nivolumab versus docetaxel in advanced squamous-cell non-small-cell lung cancer. N. Engl. J. Med. 373, 123-135. https://doi.org/10.1056/NEJMoa1504627 (2015).

4. Herbst, R. S. et al. Pembrolizumab versus docetaxel for previously treated, PD-L1-positive, advanced non-small-cell lung cancer (KEYNOTE-010): a randomised controlled trial. Lancet 387, 1540-1550. https://doi.org/10.1016/S0140-6736(15)01281-7 (2016).

5. Reck, M. et al. Pembrolizumab versus chemotherapy for PD-L1-positive non-small-cell lung cancer. N. Engl. J. Med. 375, 18231833. https://doi.org/10.1056/NEJMoa1606774 (2016).

6. Rittmeyer, A. et al. Atezolizumab versus docetaxel in patients with previously treated non-small-cell lung cancer (OAK): a phase 3, open-label, multicentre randomised controlled trial. Lancet 389, 255-265. https://doi.org/10.1016/S0140-6736(16)32517-X (2017).

7. Corrigan-Curay, J., Sacks, L. \& Woodcock, J. Real-world evidence and real-world data for evaluating drug safety and effectiveness. JAMA 320, 867-868. https://doi.org/10.1001/jama.2018.10136 (2018).

8. Miksad, R. A. \& Abernethy, A. P. Harnessing the power of real-world evidence (RWE): a checklist to ensure regulatory-grade data quality. Clin. Pharmacol. Ther. 103, 202-205. https://doi.org/10.1002/cpt.946 (2018).

9. Rothwell, P. M. External validity of randomised controlled trials: "to whom do the results of this trial apply?". Lancet 365, 82-93. https://doi.org/10.1016/S0140-6736(04)17670-8 (2005).

10. Califano, R. et al. Immune checkpoint blockade for non-small cell lung cancer: what is the role in the special populations?. Eur. J. Cancer 125, 1-11. https://doi.org/10.1016/j.ejca.2019.11.010 (2020).

11. Hurria, A. \& Kris, M. G. Management of lung cancer in older adults. CA Cancer J. Clin. 53, 325-341. https://doi.org/10.3322/canjc lin.53.6.325 (2003).

12. Al-Baimani, K. et al. Are clinical trial eligibility criteria an accurate reflection of a real-world population of advanced non-smallcell lung cancer patients?. Curr. Oncol. 25, e291-e297. https://doi.org/10.3747/co.25.3978 (2018).

13. Cramer-van der Welle, C. M. et al. Systematic evaluation of the efficacy-effectiveness gap of systemic treatments in metastatic nonsmall cell lung cancer. Eur. Respir. J. https://doi.org/10.1183/13993003.01100-2018 (2018).

14. Smit, H. J. M. et al. Effects of checkpoint inhibitors in advanced non-small cell lung cancer at population level from the National Immunotherapy Registry. Lung Cancer 140, 107-112. https://doi.org/10.1016/j.lungcan.2019.12.011 (2020).

15. Santeon Hospital Group. Feiten en ciffers 2019. https://santeon.nl/over-santeon/feiten-en-cijfers/ (2019).

16. Netherlands Comprehensive Cancer Organization. Netherlands Cancer Registry 2019. www.cijfersoverkanker.nl (2019).

17. van de Garde, E. M. W. et al. Pharmacotherapy within a learning healthcare system: rationale for the Dutch Santeon Farmadatabase. Eur. J. Hosp. Pharm. 26, 46-50. https://doi.org/10.1136/ejhpharm-2017-001329 (2019).

18. Harris, P. A. et al. Research electronic data capture (REDCap): a metadata-driven methodology and workflow process for providing translational research informatics support. J. Biomed. Inform. 42, 377-381. https://doi.org/10.1016/j.jbi.2008.08.010 (2009).

19. Guyot, P., Ades, A. E., Ouwens, M. J. \& Welton, N. J. Enhanced secondary analysis of survival data: reconstructing the data from published Kaplan-Meier survival curves. BMC Med. Res. Methodol. 12, 9. https://doi.org/10.1186/1471-2288-12-9 (2012).

20. Velcheti, V. et al. Outcomes of first-line pembrolizumab monotherapy for PD-L1-positive (TPS $>/=50 \%$ ) metastatic NSCLC at US oncology practices. Immunotherapy 11, 1541-1554. https://doi.org/10.2217/imt-2019-0177 (2019).

21. Amrane, K. et al. First-line pembrolizumab for non-small cell lung cancer patients with PD-L $1>l=50 \%$ in a multicenter real-life cohort: the PEMBREIZH study. Cancer Med. 9, 2309-2316. https://doi.org/10.1002/cam4.2806 (2020).

22. Tambo, Y. et al. Real-world efficacy of first-line pembrolizumab in patients with advanced or recurrent non-small-cell lung cancer and high PD-L1 tumor expression. Clin. Lung Cancer https://doi.org/10.1016/j.cllc.2020.02.017 (2020).

23. Crino, L. et al. Italian cohort of nivolumab expanded access program in squamous non-small cell lung cancer: results from a realworld population. Oncologist 24, e1165-e1171. https://doi.org/10.1634/theoncologist.2018-0737 (2019). 
24. Schouten, R. D., Muller, M., de Gooijer, C. J., Baas, P. \& van den Heuvel, M. Real life experience with nivolumab for the treatment of non-small cell lung carcinoma: data from the expanded access program and routine clinical care in a tertiary cancer centre-The Netherlands Cancer Institute. Lung Cancer 126, 210-216. https://doi.org/10.1016/j.lungcan.2017.11.012 (2018).

25. Grossi, F. et al. Real-life results from the overall population and key subgroups within the Italian cohort of nivolumab expanded access program in non-squamous non-small cell lung cancer. Eur. J. Cancer 123, 72-80. https://doi.org/10.1016/j.ejca.2019.09.011 (2019).

26. Martin, C. et al. Efficacy and safety of nivolumab in previously treated patients with non-small-cell lung cancer: real world experience in Argentina. Clin. Lung Cancer https://doi.org/10.1016/j.cllc.2020.02.014 (2020).

27. Spigel, D. R. et al. Safety, efficacy, and patient-reported health-related quality of life and symptom burden with nivolumab in patients with advanced non-small cell lung cancer, including patients aged 70 years or older or with poor performance status (CheckMate 153). J. Thorac. Oncol. 14, 1628-1639. https://doi.org/10.1016/j.jtho.2019.05.010 (2019).

28. Lisberg, A. et al. Treatment-related adverse events predict improved clinical outcome in NSCLC patients on KEYNOTE-001 at a single center. Cancer Immunol. Res. 6, 288-294. https://doi.org/10.1158/2326-6066.CIR-17-0063 (2018).

29. Haratani, K. et al. Association of immune-related adverse events with nivolumab efficacy in non-small-cell lung cancer. JAMA Oncol. 4, 374-378. https://doi.org/10.1001/jamaoncol.2017.2925 (2018).

30. Ricciuti, B. et al. Impact of immune-related adverse events on survival in patients with advanced non-small cell lung cancer treated with nivolumab: long-term outcomes from a multi-institutional analysis. J. Cancer Res. Clin. Oncol. 145, 479-485. https://doi.org/ 10.1007/s00432-018-2805-3 (2019).

31. Mushti, S. L., Mulkey, F. \& Sridhara, R. Evaluation of overall response rate and progression-free survival as potential surrogate endpoints for overall survival in immunotherapy trials. Clin. Cancer Res. 24, 2268-2275. https://doi.org/10.1158/1078-0432.CCR17-1902 (2018).

32. Tan, A., Porcher, R., Crequit, P., Ravaud, P. \& Dechartres, A. Differences in treatment effect size between overall survival and progression-free survival in immunotherapy trials: a meta-epidemiologic study of trials with results posted at ClinicalTrials.gov. J. Clin. Oncol. 35, 1686-1694. https://doi.org/10.1200/JCO.2016.71.2109 (2017).

33. Reck, M. et al. Updated Analysis of KEYNOTE-024: pembrolizumab versus platinum-based chemotherapy for advanced nonsmall-cell lung cancer with PD-L1 tumor proportion score of 50\% or greater. J. Clin. Oncol. 37, 537-546. https://doi.org/10.1200/ JCO.18.00149 (2019).

34. Antonia, S. J. et al. Four-year survival with nivolumab in patients with previously treated advanced non-small-cell lung cancer: a pooled analysis. Lancet Oncol. 20, 1395-1408. https://doi.org/10.1016/S1470-2045(19)30407-3 (2019).

\section{Author contributions}

E.M.W.v.d.G., F.M.N.H.S. and H.J.M.G. obtained research funding. C.M.C.v.d.W., M.V.V., M.T., B.J.M.P. and E.M.W.v.d.G. were involved in conception and study design. E.M.W.v.d.G. contributed to study supervision. C.M.C.v.d.W., M.T. and M.V.V. were responsible for acquisition of data and data analysis. C.M.C.v.d.W., M.T., B.J.M.P., F.M.N.H.S., O.H.K., H.J.M.G. and E.M.W.v.d.G. contributed to interpretation of data. C.M.C.v.d.W. and M.T. wrote the main manuscript text. All authors reviewed the manuscript.

\section{Funding}

Dutch Cancer Society (Grant Number SAN2016-7942). This funding source had no role in study design; data collection, analysis and interpretation of data; writing the report; or decision to submit the article for publication.

\section{Competing interests}

EvdG reports research grants from the Dutch Cancer Society during the conduct of the study, and from AbbVie NL outside the submitted work. HG reports a grant from Boehringer-Ingelheim and other from BMS, Roche, Novartis, Merck and Pfizer, outside the submitted work. All remaining authors have declared no conflicts of interest.

\section{Additional information \\ Supplementary Information The online version contains supplementary material available at https://doi.org/ 10.1038/s41598-021-85696-3.}

Correspondence and requests for materials should be addressed to C.M.C.d.

Reprints and permissions information is available at www.nature.com/reprints.

Publisher's note Springer Nature remains neutral with regard to jurisdictional claims in published maps and institutional affiliations.

(c) (1) Open Access This article is licensed under a Creative Commons Attribution 4.0 International (c) License, which permits use, sharing, adaptation, distribution and reproduction in any medium or format, as long as you give appropriate credit to the original author(s) and the source, provide a link to the Creative Commons licence, and indicate if changes were made. The images or other third party material in this article are included in the article's Creative Commons licence, unless indicated otherwise in a credit line to the material. If material is not included in the article's Creative Commons licence and your intended use is not permitted by statutory regulation or exceeds the permitted use, you will need to obtain permission directly from the copyright holder. To view a copy of this licence, visit http://creativecommons.org/licenses/by/4.0/.

(C) The Author(s) 2021 


\section{The Santeon NSCLC Study Group}

E. A. Kastelijn ${ }^{3}$, L. C. Vermeer ${ }^{6}$, B. E. E. M. van den Borne' ${ }^{7}$ J. W. G. van Putten ${ }^{8}$, J. H. Schouwink ${ }^{9}$ \& A. A. J. Smit ${ }^{10}$

${ }^{6}$ Department of Pulmonary Diseases, Canisius-Wilhelmina Hospital, Nijmegen, The Netherlands. ${ }^{7}$ Department of Pulmonary Diseases, Catharina Hospital, Eindhoven, The Netherlands. ${ }^{8}$ Department of Pulmonary Diseases, Martini Hospital, Groningen, The Netherlands. ${ }^{9}$ Department of Pulmonary Diseases, Medisch Spectrum Twente, Enschede, The Netherlands. ${ }^{10}$ Department of Pulmonary Diseases, OLVG, Amsterdam, The Netherlands. 